\title{
Ductus Venosus
}

National Cancer Institute

\section{Source}

National Cancer Institute. Ductus Venosus. NCI Thesaurus. Code C34143.

A vein occurring during embryonic development that is the continuation of the left

umbilical vein that drains into the inferior vena cava. 